\title{
LA MEDIACION EN LOS PROCESOS DE NEGOCIACION: UN ESTUDIO EXPERIMENTAL
}

\author{
Gonzalo Serrano \\ Maite Méndez \\ Universidad de Santiago de Compostela
}

\section{Resumen}

El presente experimento compara diversas formas de mediación entre sí y en relación con la negociación sin intervención de terceros de cada ra la resolución de conflictos sociales.

Se estudia la influencia de las condiciones de mediación y la intensidad del conflicto sobre el grado de acuerdo alcanzado, el clima y la satisfacción a lo largo de la negociación.

Se sugieren varias explicaciones con relación a los resultados obtenidos, pero las hipótesis iniciales no quedan totalmente confirmadas.

\section{Abstract}

This esperimental study compare several forms of mediation between themselves and with a simple bargaining in order to resolve social conflicts.

We have inquired into the influence of the mediation conditions and the intensity of conflict on the degree of agreement obteined, the climax and the satisfaction along the negotiation.

Some explanations for these results are suggested, but the initial hypothesis not always are confirmed.

\section{Introducción}

La mediación como forma de resolución de conflictos ha cobrado un auge muy especial en los últimos años extendiéndose a prácticamente todos los campos de la vida social en los que tienen cabida los procesos de negociación.

Pese a que la investigación referente a la mediación, su efectividad, sus determinantes, etc., se encuentra todavia en fase inicial, abundando resultados contradictorios y, en ausencia de modelos teóricos comúnmente aceptados, parece, no obstante, existir el acuerdo de que la mediación es un proceso dinámico e interactivo que viene determinado por un conjunto muy amplio de variables concerniente tanto a los sujetos implicados y sus conductas como a factores situacionales y contextuales. 
Quizá ha sido la variable "intensidad del conflicto" a la que más frecuentemente se ha recurrido para explicar resultados contradictorios respecto a la efectividad de determinadas tácticas de mediación, aduciendo que algunas de ellas son más o menos eficaces según la intensidad del conflicto que se está tratando (Rubin, 1980). Sin embargo, en muchas ocasiones, este tipo de explicación se ha buscado a posteriori, de modo que actualmente no es posible todavía establecer una relación clara entre el nivel de intensidad del conflicto y la efectividad de la mediación. Por ello nos planteamos introducir esta variable como un factor principal en nuestro diseño, con la esperanza de extraer algunas conclusiones acerca de su exacto papel.

Por otro lado, la efectividad de la mediación generalmente se ha estudiado en comparación con otras formas de resolución del conflicto, como es la negociación o diversos tipos de intervención de una tercera parte neutral; en las escasas investigaciones en las que se ha abordado directamente, los planteamientos se han centrado en tácticas o conductas de mediación muy concretas y de muy variada índole, lo cual no ha permitido concluir de modo claro para ofrecer una perspectiva general de la efectividad de lo que podríamos denominar "tipos básicos de negociación".

En base a estas sugerencias, abordaremos tanto el tema de la efectividad de la mediación en comparación con los procesos de negociación simplemente considerados, como el problema de la efectividad diferencial de distintas formas de intervención del mediador, desde una perspectiva más amplia y sin centrar la atención en tácticas concretas; los modos básicos de mediación que vamos a considerar son tres: mediación pasiva, mediación centrada en la tarea y mediación centrada en las relaciones personales; tal clasificación era la más coincidente con nuestros propósitos y, a la vez, paralela a otras de autores relevantes (Pruitt, 1981; Touzard, 1981; Kressel y Pruitt, 1985).

El carácter interactivo que se atribuye a los procesos de mediación nos hizo no sólo considerar el posible efecto principal de los dos factores que hemos definido, sino que además prestamos una especial atención a la posible actuación conjunta de ambos respecto a la efectividad de la mediación.

También el tema de la delimitación y operatividad de la "efectividad de la mediación", que lógicamente debía constituirse en variable dependiente, fue abordada como lo hace la mayoría de las investigaciones sobre el tema; es decir, en base a la tasa de acuerdos que se consiguen.

Son muchos los autores que se refieren también a aspectos tales como la satisfacción personal de los participantes, el incremento de actitudes de tipo cooperativo o competitivo, la disminución o aumento de hostilidades, etc. Por ello, hemos considerado necesario indagar no sólo el grado de acuerdo obtenido, sino también la actitud agresiva y competitiva, la tensión general y la satisfacción que mostraron los negociadores.

En resumen, se pretende una aproximación general al tema de la eficacia de la mediación. La carencia de resultados concluyentes en el estado actual de la investigación no ha permitido lógicamente el establecimiento de hipótesis muy especificas; pese a ello, consideramos conveniente formular una serie de hipótesis de tipo conceptual que permitan ordenar y estructurar la investigación.

En base, pues, al planteamiento del problema que acabamos de hacer, las hipótesis centrales serán las siguientes:

A) La mediación resulta efectiva como forma de resolución de conflictos.

B) La intensidad del conflicto influye sobre la efectividad de la mediación.

C) El tipo de mediación utilizado incidirá en la eficacia de la resolución del conflicto.

D) El tipo de mediación y la intensidad del conflicto son variables que interactúan con respecto a la eficacia, clima y satisfacción de la negociación. 


\subsection{El tema de la negociación}

En base a la literatura existente (Mc.Grath, 1962; Vidmar, 1971; Bartunek y al., 1975; Touzard, 1981) y a las exigencias de nuestro planteamiento, hemos considerado necesario que el tema elegido incluyera aspectos conocidos y que afectasen de modo directo a todos los sujetos que iban a participar en el experimento; de este modo, se sentirían suficientemente implicados en el mismo, lo que conllevaria un mayor compromiso y responsabilidad a la hora de defender sus puntos de vista; asímismo, se posibilitaría que los negociadores tuviesen una base argumental suficientemente amplia y firme (Bartunek y al., 1975).

En principio, fueron descartados aquellos temas que pudiesen afectar directamente a valores centrales o principios ideológicos de los sujetos, dado que este tipo de cuestiones dificultan la realización de concesiones, obstaculizando en gran medida el proceso de negociación (Touzard, 1981).

Por otra parte, el tema seleccionado debería ser fácilmente medible y cuantificable, pudiendo especificarse en puntos concretos sobre los cuales se iba a negociar.

Un tema que reunía tales requisitos fue "la introducción de la lengua gallega en la enseñanza universitaria en Galicia". Además de reunir todas las ventajas ya señaladas, se trata de una cuestión de actualidad, relevante y polémica. Tomamos como puntos concretos de negociación la introducción de la lengua gallega en tres ámbitos: la administración (impresos de matrícula, instancias, solicitudes, etc.), las publicaciones (tesis, artículos, apuntes, etc.) y las clases impartidas.

\subsection{Variables}

Dos fueron las variables independientes:

A) La intensidad del conflicto, descompuesta en tres niveles: conflicto alto, conflicto medio y conflicto bajo.

B) La mediación, en cuatro condiciones diferentes: ausencia de mediación, mediación pasiva, mediación centrada en la tarea y mediación centrada en las relaciones personales.

Se consideraron las siguientes variables dependientes:

A) El grado de acuerdo, que expresaba, como ya hemos dicho la efectividad del proceso.

B) La actitud competitiva mantenida por las partes a lo largo de la negociación.

C) La actitud agresiva evidenciada asímismo por los negociadores.

D) La tensión general producida a lo largo de cada sesión.

E) La satisfacción mostrada al final por los sujetos.

Consideramos necesario controlar una serie de variables, con el fin de evitar la posible contaminación experimental que pudiera producirse:

A) Intentamos neutralizar las variables edad y sexo de los sujetos. La edad se mantuvo fija para todos los negociadores; los seleccionados tenían edades comprendidas entre 19 y 20 años. Para anular las posibles influencias debidas a la diferencia de sexo escogimos para negociar únicamente a mujeres; ello se debió a que en la muestra total tomada para elegir a los negociadores el número de mujeres era bastante superior al de hombres. Con relación a los sujetos que iban actuar como mediadores, y por razones análogas pero contrapuestas a las anteriores, seleccionamos exclusivamente a sujetos masculinos.

B) Otro aspecto a controlar está relacionado con la imagen de autoridad que los 
mediadores podrían producir a priori sobre los negociadores. Por ello fueron seleccionados sujetos que no hubiesen tenido nunca contacto directo con los negociadores.

C) Se utilizaron tres mediadores diferentes, cada uno de los cuales desempeñaría uno de los tres roles que ya hemos definido. Para evitar sesgos debidos a diferencias individuales nos pareció relevante que fuese siempre la misma persona la encargada de asumir cada uno de los roles a lo largo de todas las situaciones experimentales. También señalemos que los mediadores no conocian las hipótesis del trabajo y no poseían información acerca de los otros aspectos de las negociaciones en que iban a actuar. De esta forma se podía anular influencias debidas a un posible condicionamiento.

D) Debido a la técnica de registro de información a utilizar, era necesario contar con la ayuda de jueces observadores. Fueron seleccionados tres jueces diferentes, que tenían la misión de observar todas y cada una de las sesiones. Utilizar varios jueces se debió a garantizar la fiabilidad de las medidas. Como en casos anteriores, los jueces no tenían información sobre las características de cada una de las negociaciones que iban a observar.

E) Por lo que toca a las condiciones ambientales, las sesiones se llevaron a cabo en el mismo espacio, en el cual se mantuvo constante la distribución de todos los elementos.

F) Un último aspecto a controlar era el posible intercambio de información entre los sujetos que participaban en el experimento, acerca de los incidentes y el desarrollo de la sesión. Para solucionar este problema pedíamos que se abstuvieran de realizar ningún tipo de comentario sobre lo que allí ocurría. Finalmente, se trató de combinar los negociadores de forma que previamente no se conociesen entre ellos.

\subsection{Definición de las variables}

La variable intensidad del conflicto constaba de tres niveles, cada uno de los cuales fue establecido de acuerdo a las puntuaciones obtenidas en una escala de actitudes sobre, como ya hemos dicho, "la lengua gallega y su introducción en la enseñanza universitaria en Galicia". Una vez establecidos los grupos - alto, medio, bajo- se combinaron los sujetos para poder establecer así las tres condiciones o nivel de conflicto. $\mathrm{Pa}$ ra la situación de conflicto alto, se formaron pares de sujetos, uno perteneciente al grupo alto y otro al grupo bajo, con una diferencia de puntuación de 70-85 puntos. Parece lógico que al existir tan gran diferencia el conflicto que se iba a producir sería elevado. Respecto al grupo de conflicto medio, se formaron pares de sujetos tomando uno del grupo alto y otro del grupo medio, con una diferencia de 50-45 puntos. Finalmente el grupo de conflicto bajo estaba formado por parejas de sujetos de tipo medio, existiendo entre ellos una diferencia de puntuación entre 20-25 puntos; el conflicto a producirse lógicamente debería ser de escasa intensidad dado que los sujetos iban a tener opiniones similares con relación al tema negociable.

Las condiciones de la mediación eran cuatro: una consistente en ausencia de mediador, o sea un caso simulado de negociación simple entre dos sujetos a los cuales se leía previamente el guión de la negociación. Las otras tres consistian en negociaciones entre dos sujetos con la intervención de una tercera parte que asumía para cada sesión el rol de mediador pasivo o mediador centrado en la tarea o mediador centrado en las relaciones personales; las diferencias entre estos diversos papeles son claras y suficientemente bien definidas en la literatura respectiva, por lo que, en aras de la brevedad omitimos la enumeración de las características.

La variable grado de acuerdo hace referencia al nivel de acuerdo obtenido en cada negociación con respecto a cada uno de los tres puntos del guión: administración, pu- 
blicaciones y clases. El grado de acuerdo era medido por los jueces, quienes lo puntuaban una vez finalizada la sesión en base a una escala de 5 puntos que oscilaba desde "nada" a "total", después de definir pormenorizadamente qué significado se otorgaba a cada puntuación. Aunque los jueces calificaban por separado los tres factores, para los análisis estadísticos dichas puntuaciones se unificaban en una sola.

Por actitud competitiva se entiende la resistencia de los negociadores a realizar concesiones, al tiempo que intentaban forzar la postura de la parte contraria. Las puntuaciones a dicha actitud se realizaron como en el caso anterior.

La actitud agresiva hace referencia a la conducta mostrada por cada negociador al defender sus puntos de vista: alteraciones del tono de voz, insultos, amenazas, etc. El sistema de medida era asimismo análogo a los casos precedentes.

Se entiende por tensión general el ambiente general en que se desarrollaba cada sesión: enfrentamientos entre las partes, situaciones limite, posturas opuestas muy agudizadas, etc. La forma de puntuación era también similar a las anteriores.

La satisfacción por la que se preguntaba a los negociadores englobaba los siguientes aspectos: el grado de satisfacción personal con respecto a los resultados obtenidos, los sentimientos que inspiraba la otra parte y la actitud percibida del negociador opuesto con respecto a uno mismo.

\section{METODO}

\subsection{Muestra}

Los negociadores participantes en el experimento voluntariamente fueron $48 \mathrm{mu}$ jeres, alumnas de varias secciones de la Facultad de Filosofía y Ciencias de la Educación de la Universidad de Santiago de Compostela.

La selección se llevó a cabo en base a las puntuaciones obtenıdas por cada unia de ellas en la escala de actitudes ya mencionada y en la escala Assertion Inventory (A.I.) de Gambrill-Richey, (1975). Para completar las parejas de negociadores, se requería 16 sujetos pertenecientes al grupo A (inclinación más positiva), 8 sujetos pertenecientes al grupo B (orientación negativa) y 24 sujetos del grupo $\mathrm{N}$ (inclinación media-neutral). Por otra parte, los 48 sujetos deberian haber obtenido una puntuación en la escala de asertividad inferior a 140 puntos, tomándose la puntuación 200 como "nada asertivo" y la puntuación 31 como "muy asertivo", siendo éstas las puntuaciones extremas; parecía necesario asegurar que los sujetos participantes serian lo suficientemente asertivos como para defender sus propios puntos de vista.

La selección de los sujetos que habían de actuar ocmo mediadores se llevó a cabo entre varios individuos que se adaptaban al perfil que el experimento exigia; es decir, varones y personas desconocidas para los negociadores.

Estas personas fueron sometidas a diferentes sesiones de role-playing para poder comprobar la adecuación de cada uno cara al desempeño del rol de mediador. En base a sus actuaciones, un grupo de expertos seleccionó a los 3 de ellos que mejor se ajustaban a las características de la mediación pasiva, la mediación centrada en la tarea.y la mediación centrada en las relaciones personales. Posteriormente, se realizaron nuevas sesiones de role-playing con el fín de que los futuros mediadores tomasen experiencia del papel que deberian desempeñar.

Finalmente fueron seleccionados 3 jueces, a los que se les explicó en que consistía su función y con los que se realizaron sesiones de entrenamiento con el fin de que se familiarizasen con los instrumentos de registro y la situación experimental.

\subsection{Diseño Experimental}

Nos planteamos un diseño experimental $3 \times 4$ intersujetos. El primer factor (A), 
intensidad de conflicto, es un factor intersujeto y presenta 3 niveles (conflicto alto, conflicto medio, conflicto bajo). El segundo factor (B), mediación, es también un factor intersujeto con 4 niveles (sin mediador, mediador pasivo, mediador centrado en la tarea, mediador centrado en las relaciones personales).

Se realizaron dos pases de cada una de las condiciones experimentales, siendo, por tanto, 24 el número total de sesiones realizadas. El tiempo de duración de cada una fué de 25 minutos de los cuales 15 correspondían a la negociación propiamente dicha; en el tiempo restante, se les leía a los sujetos el guión de la negociación y cubrían los cuestionarios de satisfacción.

\subsection{Instrumentos de medida, materiales utilizados y procedimiento.}

Para la realización del experimento ha sido necesario utilizar los siguientes materiales:

- Escala de Actitudes sobre la introducción de la lengua gallega en la universidad, construída por nosotros mismos, según el método de Likert.

- El "Assertion Inventory" de Gambrill y Richey (1975).

- Hoja de Instrucciones para los mediadores, dónde se explicaba las características especificas del rol a desempeñar.

- Texto previo a la negociación en que se explicaba a los negociadores el sentido de la prueba dando las instrucciones oportunas y necesarias para el desarrollo de la misma.

- Guión de la negociación, donde quedaban señalados los puntos en base a los cuales se iba a negociar.

- Cuestionarios de Satisfacción Individual. realizar.

- Hoja de Registro utilizada por los jueces para las distintas puntuaciones a

La aplicación y realización del diseño se llevó a cabo en dos fases. En la primera de ellas se procedió a seleccionar el tema y a elaborar la escala de actitudes con que seleccionar a los negociadores. El paso siguiente fue la elaboración del material antes reseñado. Posteriormente, se realizó el proceso de selección y entrenamiento de mediadores y jueces en la forma expuesta.

La segunda fase, experimental propiamente dicha, se desarrolló teniendo presente que, debido a los cuatro niveles de la variable mediación, se requería tener dos tipos de sesiones experimentales de diferente formato, una para el nivel sin mediador y otra para los restantes en que el mediador estaba presente.

La secuencia del experimento puede resumirse en los siguientes pasos:

- Introducción de los sujetos en el laboratorio y ocupación de los jueces de su lugar de observación (por supuesto, no visible para los negociadores).

- Lectura a los negociadores del texto y guión de la negociación por parte del experimentador o del mediador, según el caso.

- Negociación.

- Cumplimentación de los cuestionarios de satisfacción.

- Cumplimentación por parte de los jueces de las hojas de registro.

\subsection{Análisis de datos}

Un requisito imprescindible antes de proceder a la aplicación de las pruebas estadísticas e interpretación de los datos obtenidos consistía en verificar la homogeneidad en las puntuaciones otorgadas por los jueces; para ello utilizamos el Coeficiente de Concordancia de Kendall, aplicado a las 192 puntuaciones que cada uno de los tres jueces emitió a lo largo del experimento. 
El siguiente objetivo era comprobar si existian diferencias sobre el grado de acuerdo alcanzado en cada uno de los tres temas a negociar (administración, publicaciones y clases). Desde el comienzo se pensó que el aspecto "administración" sería más fácil para obtener acuerdos, seguido de "publicaciones" y, por último, "clases"; para comprobarlo se aplicó un análisis trifactorial mixto con dos factores intersujeto (intensidad de conflicto y tipo de mediación) y un factor intrasujeto (temas de negociación).

Una vez constatado, debia probarse si las diferencias establecidas venían dadas por los efectos de las variables independientes y no por otras variables; realizamos una prueba de ponderación inversamente proporcional, con el fín de homogeneizar los resultados $\mathrm{y}$, en base a estas puntuaciones ponderadas, se llevaron a cabo los siguientes análisis estadísticos.

En primer lugar, y con objeto de hallar si la intensidad del conflicto y el tipo de mediación influían sobre el grado de acuerdo, los datos fueron sometidos a un análisis de varianza para un diseño de dos factores de niveles fijos.

Para poder conocer qué niveles de intensidad de conflicto y qué tipo de mediación resultan más efectivos se acudió a la prueba de Scheffé. Posteriormente, y para comprobar bajo qué tipo de mediación se obtenía mayor acuerdo en cada nivel de intensidad de conflicto, se utilizó la prueba $T$ de Student.

Las puntuaciones correspondientes a las actitudes agresiva y competitiva, la tensión general y la satisfacción fueron tratadas con los mismos estadisticos.

\section{RESULTADOS}

1. Se confirma la homogeneidad de las puntuaciones de los jueces al no haber diferencias significativas entre ellas $\left(W=.017 ; X^{2}=9.74 ;\right.$ p. «.01).

2. Tanto el factor "conflicto" como el factor "mediación" determinan significativamente el grado de acuerdo (Cfr. Tabla 1).

TABLA 1

\begin{tabular}{|c|c|c|c|c|}
\hline $\begin{array}{l}\text { FUENTES DE } \\
\text { VARIACION }\end{array}$ & $\begin{array}{c}\text { SUMA } \\
\text { DE } \\
\text { CUADRADOS }\end{array}$ & $\begin{array}{c}\text { GRADOS } \\
\text { DE } \\
\text { LIBERTAD }\end{array}$ & $\begin{array}{c}\text { MEDIA } \\
\text { DE } \\
\text { CUADRADOS }\end{array}$ & $\mathbf{F}$ \\
\hline FACTOR CONFLICTO (A) & 43556,31 & 2 & 21778,15 & $11,95 * *$ \\
\hline FACTOR MEDIACION (B) & 56244,93 & 3 & 18748,31 & $10,29 * *$ \\
\hline INTERACCION $(\mathrm{A} \times \mathrm{B})$ & 14640,79 & 6 & 2440,13 & 1,34 \\
\hline INTRAGRUPO & 109269,89 & 60 & 1821,16 & \\
\hline TOTAL & 223711,92 & 71 & & \\
\hline
\end{tabular}


2.1. En la condición "sin mediador" (SM) se alcanza mayor acuerdo que en la condición "mediador pasivo" (MP) $(\alpha=0.05)$.

La condición "mediador centrado en la tarea" (MCT) es más positiva de cara al acuerdo que MP $(\alpha=0.01)$.

MCT permite un menor acuerdo que la "mediación centrada en las reluciones personales"' (MCR) $(\alpha=0.01)$.

2.2. Con relación al grado de acuerdo, aparecen diferencias significativas entre conflicto alto (CA) y conflicto medio (CM) o conflicto bajo (CB), de modo que el primero lo hace significativamente menor $(\alpha=0.01)$. No se hallaron diferencias entre $\mathrm{CM}$ y $\mathrm{CB}$.

2.3. En situaciones de CA, comparando todas las condiciones entre sí, la MCR es significativamente más positiva que las demás. Las restantes comparaciones no presentan diferencias significativas.

En situaciones de CM, acontece igual que en el caso anterior, pero ahora con la MCT.

En situaciones de $\mathrm{CB}$, la MP es significativamente más negativa para el acuerdo que el resto.

3. Con relación a la actitud competitiva los datos evidencian que sobre ella influye significativamente tanto la intensidad del conflicto como el modo de mediación ( $\mathrm{Cfr}$. Tabla 2).

TABLA 2

\begin{tabular}{|c|c|c|c|c|}
\hline $\begin{array}{l}\text { FUENTES DE } \\
\text { VARIACION }\end{array}$ & $\begin{array}{c}\text { SUMA } \\
\text { DE } \\
\text { CUADRADOS }\end{array}$ & $\begin{array}{l}\text { GRADOS } \\
\text { DE } \\
\text { LIBERTAD }\end{array}$ & $\begin{array}{c}\text { MEDIA } \\
\text { DE } \\
\text { CUADRADOS }\end{array}$ & $\mathbf{F}$ \\
\hline FACTOR CONFLICTO (A) & 16,08 & 2 & 8,04 & $20,61 * *$ \\
\hline FACTOR MEDIACION (B) & 2,971 & 3 & 2,99 & $7,66^{* *}$ \\
\hline INTERACCION $(\mathrm{A} \times \mathrm{B})$ & 3,799 & 6 & 0,63 & 1,61 \\
\hline INTRASUJETO & 14,28 & 36 & 1,61 & \\
\hline TOTAL & 43,13 & 47 & & \\
\hline
\end{tabular}

3.1. Tomando como referencia únicamente el tipo de mediación, la condición MP genera una mayor actitud competitiva que las otras modalidades. También la MCR posibilitaba más la competitividad que la MCT $(\mathrm{t}=3.05 ; \mathrm{p} .<.05)$.

3.2. Respecto al̈ nivel de conflicto, el CA, de modo significativo, ocasionaba un mayor índice de competitividad. 
3.3. Teniendo en cuenta el nivel de intensidad del conflicto y el tipo de mediación utilizado, los resultados significativos son los siguientes:

En situaciones de CA las condiciones de SM y MP parecen las más proclives a generar actitudes competitivas. Sin embargo, la MCT se muestra, por el contrario, la más positiva.

Bajo $\mathrm{CM}$, las únicas comparaciones significativas fueron entre SM y MCR por una parte, y entre MCR y MCT, comprobándose en ambos casos que la MCR generaba mayor tasa de competitividad.

En condiciones de CB, la MP produce mayor actitud competitiva que el resto de tipos de mediación.

4. En lo tocante a la actitud agresiva, de nuevo resulta positiva la influencia tanto del conflicto como de la mediación (Cfr. Tabla 3).

TABLA 3

\begin{tabular}{|l|c|c|c|c|}
\hline \multicolumn{1}{|c|}{ FUENTES DE } & $\begin{array}{c}\text { SUMA } \\
\text { DE } \\
\text { VARIACION } \\
\text { CUADRADOS }\end{array}$ & $\begin{array}{c}\text { GRADOS } \\
\text { DE } \\
\text { LIBERTAD }\end{array}$ & $\begin{array}{c}\text { MEDIA } \\
\text { DE } \\
\text { CUADRADOS }\end{array}$ & F \\
\hline FACTOR CONFLICTO (A) & 11,12 & 2 & 5,56 & $21,38 * *$ \\
FACTOR MEDIACION (B) & 7,8 & 3 & 2,36 & $9,07 * *$ \\
INTERACCION (A x B) & 2,78 & 6 & 0,46 & 1,76 \\
INTRAGRUPO & 9,57 & 36 & 0,26 & \\
\hline TOTAL & & 47 & & \\
\hline
\end{tabular}

4.1. La condición de MP era más proclive a la agresión que las de MCT y MCR.

4.2. De nuevo, en situación de CA se daba una mayor agresividad que en el resto de situaciones conflictivas.

4.3. Considerando ahora los dos factores (conflicto y mediación), tenemos los siguientes resultados:

Solamente, en CA, la MP se mostraba como más posibilitadora de agresividad que la MCT $(\mathrm{t}=3.43, \mathrm{p} .<.05)$.

En situaciones de CM no se hallaron diferencias significativas.

En situaciones de CB, la MP en todos los casos generaba una actitud más agresiva. 
5. Con relación a la tensión general observada a lo largo de las negociaciones, los datos son coincidentes en todos sus términos con los obtenidos al referirnos a la actitud agresiva ( $\mathrm{Cfr}$. Tabla 4).

TABLA 4

\begin{tabular}{|c|c|c|c|c|}
\hline \multirow{2}{*}{ FUENTES DE } & $\begin{array}{c}\text { SUMA } \\
\text { DE } \\
\text { VARIACION }\end{array}$ & $\begin{array}{c}\text { GRADOS } \\
\text { DE } \\
\text { CIAADRADOS }\end{array}$ & $\begin{array}{c}\text { MEDIA } \\
\text { DE } \\
\text { CUADRADOS }\end{array}$ & F \\
\hline FACTOR CONFLICTO (A) & 6,82 & 2 & 3,41 & $16,23 * *$ \\
FACTOR MEDIACION (B) & 4,74 & 3 & 1,58 & $7,52 * *$ \\
INTERACCION (A $\times$ B) & 1,44 & 6 & 0,24 & \\
INTRAGRUPO & 2,57 & 12 & 0,21 & \\
\hline TOTAL & 17,57 & 23 & & \\
\hline
\end{tabular}

6. Finalmente, los resultados concernientes a la satisfacción mostrada después del proceso negociador (Cfr. Tabla 5).

TABLA 5

\begin{tabular}{|l|c|c|c|c|}
\hline \multicolumn{1}{|c|}{ FUENTES DE } & $\begin{array}{c}\text { SUMA } \\
\text { DER } \\
\text { VARACION }\end{array}$ & $\begin{array}{c}\text { GRADOS } \\
\text { DE } \\
\text { LIBERTAD }\end{array}$ & $\begin{array}{c}\text { MEDIA } \\
\text { DE } \\
\text { CUADRADOS }\end{array}$ & F \\
\hline FACTOR CONFLICTO (A) & 18,16 & 2 & 9,08 & $56,75 * *$ \\
FACTOR MEDIACION (B) & 1,42 & 3 & 0,47 & $2,93 *$ \\
INTERACCION (A $\times$ B) & 9,39 & 6 & 1,56 & $9,75 * *$ \\
INTRAGRUPO & 5,76 & 36 & 0,16 & \\
\hline TOTAL & & & & \\
\hline
\end{tabular}


Se constata la incidencia de las variables independientes tomadas globalmente. 6.1. Como era de esperar, a menor nivel de conflicto la satisfacción es mayor.

6.2. Las situaciones SM y MCT resultaban, en comparación con las otras, las más satisfactorias.

\section{DISCUSION}

La interpretación de los resultados obtenidos por los análisis estadísticos de los datos vamos a hacerla lógicamente en función de las cuatro hipótesis planteadas.

La primera hipótesis se refería a la efectividad de la mediación como forma de resolución de conflictos, según el grado de acuerdo alcanzado en las negociaciones. $\mathrm{Pa}$ ra verificar esta hipótesis comparamos el efecto producido por la condición sin mediador con el efecto producido por las tres condiciones de mediación.

En el análisis de varianza se observó que la variable "tipo de mediación” devenía un factor determinante del grado de acuerdo obtenido.

Ahora bien, la aplicación de la prueba de Scheffé puso de manifiesto que no existía una significativa superioridad en las tres condiciones de mediación frente a la condición sin mediación, respecto al grado de acuerdo alcanzado. De hecho, como ya indicamos, únicamente la comparación entre la condición sin mediador y la condición mediador pasivo es significativa, obteniéndose mayor grado de acuerdo con la primera.

Ante este estado de cosas, se podría alegar que puesto que las otras tres condiciones son igualmente eficaces de cara a la obtención de acuerdos, razones de tipo práctico aconsejarían el uso de una negociación sin mediador. Estos resultados no sólo cuestionan nuestra hipótesis inicial sino también las conclusiones de otros estudios sobre el tema, por lo que consideramos indispensable profundizar en el análisis de este punto.

Nos planteamos así la posibilidad de que, en concordancia con la tercera vía explicativa de Rubin (1980), la introducción de un mediador fuese efectiva en condiciones de conflicto alto y considerada como una intromisión en condiciones de conflicto medio y bajo. Para verificar tal suposición, utilizamos los resultados obtenidos de la correspondiente prueba $\mathrm{T}$ de comparación de las cuatro condiciones de mediación para cada nivel de intensidad de conflicto; estos resultados indican que únicamente existen diferencias significativas entre la condición sin mediador y mediación centrada en las relaciones personales para un nivel de conflicto alto, siendo el grado de acuerdo mayor para el segundo que para el primero.

Por lo tanto, aunque sólo sea para un tipo de mediación, la suposición de Rubin parece confirmarse. De hecho, la falta de eficacia de la mediación pasiva en situaciones de conflicto alto no es de extrañar, puesto que, en general, son las intervenciones activas y controladoras las que resultan efectivas (Pruitt-Johnson, 1970; Vidmar, 1971; Bartuneck y al., 1975; Rubin, 1980).

Más difícil de explicar parece ser, en principio, el fracaso de la mediación centrada en la tarea, que, pese a ser una intervención más controladora que la simple negociación o la mediación pasiva, no consiguió en la condición de conflicto alto un grado de acuerdo significativamente mayor que éstas. Ahora bien, de nuevo Rubin, al tratar el tema de la interacción entre la intensidad del conflicto y la efectividad de ciertas técnicas de mediación, señala un elemento que podría ayudar a la interpretación: ciertas tácticas devienen ineficaces, e incluso contraproducentes, cuando el nivel de conflicto es alto. Varios estudios (Erickson, 1974; Johnson, 1977) evidencian que otra táctica tipica de mediación centrada en la tarea, como es la identificación de los ítems o puntos a negociar, parece seŕ negativa cuando el conflicto es alto, generando grados de acuerdo menores; tal hecho puede deberse a que dicha identificación sirve en realidad para 
recordar a los sujetos el desacuerdo existente y las escasas posibilidades de alcanzar una solución satisfactoria.

Pasando a las restantes variables dependientes, numerosos autores han señalado que una de las ventajas de la mediación consiste en mejorar las relaciones entre las partes, produciendo actitudes cooperativas más que competitivas y evitando hostilidades y tensiones (Peters, 1952; Kerr, 1954; Rubin, 1980; Pruitt, 1981).

Nuestros resultados, sin embargo, repiten para las tres condiciones que estamos considerando exactamente el mismo esquema que encontrábamos para la variable grado de acuerdo. Parece ser, por tanto, que atendiendo a lo que podríamos denominar el clima general de la negociación, es preferible la no intervención de un mediador a la de un mediador pasivo, y resulta indiferente optar por una negociación sin mediador o con mediador centrado en la tarea o centrado en las relaciones personales.

En resumen, nuestros resultados no inclinan la balanza tan claramente a favor de la mediación respecto a la simple negociación. Ahora bien, no podemos decir que nuestra investigación sea la excepción; Kressel y Pruitt (1985) señalan la existencia de resultados francametne contradictorios en torno a las citadas ventajas de la mediación.

Por otra parte, varios autores (Kochan y Jick 1978; Sprenkle y Storn, 1983; Carnevale y Pegnetter, 1985; Hiltrop, 1985) apuntan la importancia de la motivación de las partes para alcanzar un mayor acuerdo. En este sentido, cabría pensar que los negociadores participantes quizá no se encontrasen lo suficientemente motivados por tratarse de una negociación simulada, en la que los resultados obtenidos no tendrían consecuencias prácticas inmediatas para ellos.

También es posible que la eficacia de la mediación deba vincularse al momento que puede atravesar una negociación, de tal manera que habría que utilizar la mediación como forma de resolver ciertas secuencias concretas y difíciles dentro del proceso de negociación.

Pasaremos seguidamente a la discusión de los resultados referidos a la segunda hipótesis, alusiva a la influencia del conflicto cara a la efectividad de la mediación.

En el análisis de varianza se pudo comprobar que la variable conflicto aparece como muy influyente en el grado de acuerdo alcanzado en la negociación. Algunos autores hacen referencia a la eficacia de la mediación en función de la situación de conflicto que se está dando (Houlden y al., 1978; Rubin, 1980; Wall, 1981).

Observando los resultados de la prueba de Scheffé para los niveles de conflicto, nos encontramos con que existen diferencias significativas entre el conflicto alto y el bajo, aconteciendo también en este caso que es en situación de conflicto alto cuando se produce un menor acuerdo. Los resultados se ven apoyados por la mayoría de estudios al respecto, pues obviamente resulta más difícil alcanzar acuerdos cuando la situación conflictiva es elevada.

Esta mayor influencia diferencial no sólo se produce en el grado de acuerdo sino que se repite también para las actitudes competitivas, agresivas y para la tensión general; si el conflicto es alto, los negociadores mantendrán posiciones de competitividad, conductas más agresivas y se provocará una mayor tensión en la negociación.

La tercera hipótesis plantea la influencia de la variable "tipo de mediación" sobre la efectividad del proceso de resolución del conflicto.

El análisis de varianza señala que el factor "tipo de mediación" ejerce un efecto significativo sobre el grado de acuerdo. Este resultado concuerda con gran parte de la literatura al respecto (Chemers y al., 1966; Touzard, 1981).

La aplicación de la prueba de Scheffé para los distintos niveles de la mediación nos ha permitido establecer diferencias entre los mismos, e incluso apuntar una cierta ordenación. 
Con relación al grado de acuerdo, se encontraron diferencias significativas en las comparaciones que incluyen la mediación centrada en la tarea, siendo el grado de acuerdo superior en tal condición. Es decir, la mediación pasiva y la mediación centrada en las relaciones personales son prácticamente igual de efectivas respecto al grado de acuerdo y resultan menos eficaces que la mediación centrada en la tarea.

Parece lógico que sea la intervención centrada en la tarea la más efectiva; como Kressel y Pruitt (1985) apuntan, el objetivo fundamental de este tipo de intervenciones es acercar las posturas y precipitar el acuerdo; además, con frecuencia, la mediación centrada en la tarea cumple un papel que otras modalidades de intervención son incapaces de satisfacer: centrar la discusión sobre los puntos relevantes y permitir realizar concesiones que de otra forma no se harían.

La actitud agresiva y la tensión general en casos de mediación pasiva son significativamente superiores. En una situación en que los sujetos se encuentran de por sí relativamente inseguros y ansiosos, la presencia de un juez vigilante de todas sus acciones no hace sino incrementar la tensión general. En cualquiera de las otras dos formas de mediación, los negociadores se enfrentaban a un sujeto activo que, de una forma u otra, les ofrecía un marco de referencia en el que desenvolverse, disminuyendo así la ansiedad y el sentimiento de indefensión.

Los resultados referentes a la actitud competitiva ofrecen un perfil ligeramente distinto; en este caso, las diferencias significativas se establecen de nuevo entre la mediación centrada en la tarea y las otras dos condiciones, produciendo estas últimas una mayor actitud competitiva. Parece lógico pensar que en la mediación pasiva y en la mediación centrada en las relaciones personales, donde el mediador no hacía referencia al contenido argumental de la negociación, los sujetos se sentían únicos defensores de su punto de vista frente al oponente, y sólo de su poder de convicción o su superioridad sobre el otro dependía que el acuerdo resultase más favorable a su postura.

En resumen, los resultados sugieren que la mediación centrada en la tarea es, a nivel general, la más ventajosa, y la mediación pasiva la menos eficaz; ésta también resulta ser significativamente menos fecunda que la negociación sin mediador.

La cuarta hipótesis suponía una interacción existente entre los efectos de las dos variables independientes, intensidad del conflicto y tipos de mediación.

En el análisis de varianza la interacción de los dos factores, sobre el grado de acuerdo, no resulta significativa; por tanto no existen combinaciones fijas de estas dos variables que produzcan un grado de acuerdo determinado; es decir, la incidencia de las dos variables se da independientemente. No se encontraron tampoco interacciones significativas referentes a la competitividad, agresividad y tensión general.

Si bien es cierto que la evidencia empírica a favor de la relevancia de la intensidad del conflicto como determinante de la eficacia de la mediación es sólida, parece menos cierto aceptar que sea el único factor que esté influyendo en los resultados de un proceso de mediación; y, consiguientemente, es posible que existan otras variables menos conocidas que estén interactuando, oscureciendo así la relación que se pretende establecer entre estos dos factores considerados centrales.

En este sentido, queremos destacar una variable, que podríamos denominar "momento del proceso", a la que sólo muy recientemente se le está otorgando la atención que, creemos, merece. Si se acepta que la mediación es fundamentalmente un proceso dinámico, parece lógico pensar que el "cuándo" se usa una táctica puede ser tan importanten como las "circunstancias" no únicamente temporales (Cfr. Kressel-Pruitt, 1985). Es decir, resulta posible que la efectividad del uso de una forma de mediación determinada dependa tanto de la intensidad del conflicto que se está manejando como del momento concreto del proceso conflictivo en que nos encontremos. 
Un conflicto no viene exclusivamente determinado, aunque sea un factor importante, por su intensidad, sino que se trata de un proceso complejo que precisa un número más amplio de variables para definirlo. En este sentido, opinamos que con una aproximación multivariada en la que se tuviesen en cuenta los efectos simultáneos de una alta gama de variables sería posible encontrar interacciones significativas que permitieran una correcta explicación del complejo problema al que nos enfrentamos.

Como hemos planteado, las diversas modalidades de mediación generan consecuencias de muy diversa índole; unas, quizá las primariamente más relevantes, están vinculadas a la resolución del conflicto como tal y expresan, por tanto, la eficacia de la fórmula mediadora. Otro tipo de consecuencias conforman lo que hemos venido llamando el clima. Variables tales como la agresividad o la tensión poseen una enorme importancia para el desarrollo de la negociación y, en aquellos casos en que ésta continúe más allá de resoluciones parciales, el mantenimiento de un clima aceptable se torna imprescindible para la continuidad positiva del proceso. Pero, además de la eficacia general y del clima interpersonal creado, es necesario tener en cuenta otra variable situada a nivel individual, que hemos denominado "satisfacción". Esta variable viene definida por la positividad de las actitudes entre las partes, el mantenimiento de una percepción positiva, la presencia de un "trust" aceptable, y por la sensación de positividad general emanada de los resultados y del proceso.

Nuestros datos muestran que, globalmente, la satisfacción viene determinada por la modalidad de mediación empleada.

Desde un punto de vista más concreto, cabe observar que una situación sin mediador no difiere significativamente del resto de tipos de mediación, salvo con la mediación pasiva, que genera el más alto grado de insatisfacción. Las conclusiones deben apuntar a la insatisfacción generada por la mediación pasiva en todos los casos y a la imposibilidad de decidir entre la negociación o alguna forma de mediación, con respecto, siempre, a la satisfacción; será preciso, de nuevo, considerar otro tipo de factores que vayan definiendo las fuentes de la variabilidad en la aparición de la satisfacción personal.

Teniendo en cuenta el conflicto como variable moduladora de la satisfacción, también globalmente aparece con una determinación positiva; la situación de conflicto alto, sea cual sea el tipo de mediación, produce una sensación de manifiesta insatisfacción en los negociadores.

\section{REFERENCIAS BIBLIOGRAFICAS}

Bartunek, J.M. et al (1975): "Third intervention and the bargaining behavior at group representatives". Journal of Conflict Resolution, 19, 532-557.

Brett, J.M. y Goldberg, S.M. (1983): "Mediator-advisors: A new third-party role". En M.H. Bazerman y R.J. Lewicki (Eds.). Negotiation in organizations, Beverly Hills: Sage.

Brown, B.R. (1977): "Face-saving and face-restoration in negotation". En D. Druckman (ed.). Negotiations: Social-Psychological perspectives. Beverly Hills, California: Sage.

Carnevale, P. y Pegnetter, R. (1985): “The Selection of mediation tactics in public sector disputes: A contingency analysis". Journal of social Issues, 41, (2), 65-81.

Chemers, M.m. et col. (1966): "Source effects of cultural training on leadership in heterocultural task groups". Intern. J. of Psychol. 1, 257-270.

Deutsch, M. (1980): "Fifty Years of Conflicti". En L. Festinger. (Ed.). Retrospections in Social Psychology. Oxford Univ. Press, New York. 
Eiseman, J.H. (1977): "A Third party consultation model for resolving recurring conflicts collaborativity". Journal of Applied Behavioral Science, 13, 303-314.

Erickson, B. et al. (1974): "Functions of a third party in the resolution of conflict: The role of a judge in petrial conferences". Journal of personality and social psycho$\log y, 30,293-306$.

Fischer, R.J. (1972): "Third party consultation: A method for the study and resolution of conflict". Journal of Conflict Resolution, 16, 67-94.

Hiltrop, J.M. (1985): "Mediator behavior and the settlement of collective bargaining disputes in Britain". Journal of Social Issues, 41, (2), 83-99.

Houlden, D. et al. (1978): "Preference for modes of dispute resolution as a function of process and decision control". Jr. of Exp. Soc. Psych., 14, 13-30.

Karim, A. y Pegnetter, R. (1983): “Mediator strategies and qualities and mediation effectiveness". Industrial Relations, 22, 105-114.

Kerr, C. (1954): "Industrial conflict and its mediation". American Journal of Socio$\log y, 60,230-245$.

Kochan, T.A. y Jick, T. (1978): "The public sector mediation process: A theory and empirical examination". Journal of Conflict Resolution, 22, 209-240.

Kressel, K. (1972): "Labor mediation: An exploratory survey". Albany, New York: Association of Labor mediation Agencies.

Kressel, K. y Pruitt, D.G. (1985): "Themes in the Mediation of Social Conflict". Journal of Social Issues, 41, 2, 179.

Lindskold, S. (1978): "Trust Development, The Grit proposal, and the Effects of Conciliatory Acts on Conflict and Cooperation". Psychological Bulletin, 85, 772-793.

McGrath, J.E. y Julian, J.W. (1962): "Negotiation and Conflict. An Experimental Study". Res. Lab. Univ. of Illinois, Urbana.

Morley, I.E. y Stephenson, G.M. (1977): The Social Psychology of Bargaining. Allen and Unwin: London.

Peters, E. (1955): Strategy and Tactics in Labor Negotiations. New London, Conn.: National Foremen's Institute.

Pruitt, D.G. (1981): Negotiation Behavior. New York: Academic Press.

Pruitt, D.G. (1983): "Strategic Choice in Negotiation". Amer. Behav. Scientist, 27, 2, 167-194.

Pruitt, D.G. y Johnson, D.F. (1970): "Mediation as an aid to face saving in negotiation". Journal of Personality and Social Psychology, 14, 239-246.

Pruitt, D.G. y Kressel, K. (1985): “The mediation of social conflict”. Jr. of Soc. Issues, $41,2,1-10$.

Rubin, J.Z. (1980): "Experimental Research on third-party intervention in conflict: Toward some generalizations". Psychological Bulletin, 87, 2, 379-391.

Rubin, J.Z. y Brown, B.R. (1975): The Social Psychology of Bargaining and Negotiation. New York: Academic Press.

Serrano, G. (1985): "Procesos de Negociación e Interacción Social”. I Congreso Nacional de Psicología Social. Granada, 1985.

Sprenkle, D.M. y Storn, C.L. (1983): "Divorce Therapy Outcome Research: A Sustantive and Methodological Review". Journal of Marital and Family Therapy, 9, 239-258.

Touzard, H. (1981): La Mediación y la Solución de Conflictos. Herder, Barcelona.

Tysoe, M. (1981): "Bargaining and Negotiation". En Colman (ed.): Cooperation and Competition in Humans and Animals. Academic Press, New York. 
Vidmar, N. (1971): "Effects of Representational Roles and Mediators on Negotiation Effectiveness". Journal of Personality and Social Psychology, 17, 48-58.

Wall, J.A. (1981): "Mediation: An Analysis, Review and Proposed Research". Journal of Conflict Resolution, 25, 157-180.

Wallton, R.E. y McKersie, G.B. (1965): A Behavioral Theory of Labor Negotiation. NcGraw Hill, New York. 